\title{
Transportation of lymph node biopsy specimens in selective Kirchner's liquid medium for culture of tubercle bacilli
}

\author{
VANAJAKUMAR, N. SELVAKUMAR, M. S. JAWAHAR, K. RAJARAM and C. N. PARAMASIVAN \\ Tuberculosis Research Centre, Mayor V.R. Ramanathan Road, Chetput, Madras-600 031, India
}

\begin{abstract}
Lymph node biopsy specimens, obtained from 297 paediatric and adult patients with tuberculous lymphadenitis at Madurai, were transported in selective Kirchner's liquid medium (KL-T) to the Tuberculosis Research Centre, Madras and processed for culture. Mycobacterium tuberculosis was isolated from $201(68 \%)$ specimens. Of the 192 specimens received within 4 days of resection, $134(69.8 \%)$ yielded $M$. tuberculosis on culture and of the 105 specimens received after 5 days, $67(63.8 \%)$ were culture positive; the difference was not statistically significant. By incubating KL-T alone further, after removing the gland for processing, it was found that mere contact with the excised node during transportation was enough to retrieve $77(38.3 \%)$ of the total of 201 positive isolates obtained, the delay did not affect the culture positivity rate. Thus, lymph node specimens for culture of tubercle bacilli can be stored in the refrigerator for up to 15 days and transported in KL-T at ambient temperature for 18-20 h without any loss in culture positivity.
\end{abstract}

\section{Introduction}

Among the extrapulmonary manifestations, tuberculous lymphadenitis is the most frequently encountered form of tuberculosis $[1,2]$. The isolation of tubercle bacilli from lymph node biopsy specimens remains the confirmatory test for the diagnosis of the disease. As lymph node specimens are usually obtained only once, they must be examined as thoroughly as possible to give the maximum chance of isolating mycobacteria [3]. Lymph node specimens are usually processed within $24 \mathrm{~h}$ of the receipt of the specimens, following the established culture procedure with multiple media [4].

The chances of recovering tubercle bacilli from the nodes recede appreciably if the interval between resection and receipt of the node in the laboratory exceeds 4 days $[5,6]$. Therefore, whenever there is unavoidable delay during transit it becomes necessary to provide a congenial milieu for tubercle bacilli present in these specimens. This study reports observations on the advantage of transporting lymph node biopsy specimens in selective Kirchner's liquid medium (KL-T).

Received 19 June 1996; accepted 13 Aug. 1996. Corresponding author: Dr C. N. Paramasivan.

\section{Material and methods}

The centre conducted a controlled clinical trial on treatment of lymph node tuberculosis, in collaboration with the paediatric and adult surgery department of the Government Rajaji Hospital, Madurai.

\section{Lymph node specimens}

A total of 297 biopsy specimens obtained from patients with tuberculous lymphadenitis diagnosed either by histopathological evidence or by bacteriological confirmation were included in this study. There were 116 children $(<12$ years) and 181 adults; 100 were males and 197 females.

\section{Transportation of specimens}

The lymph node specimens, on resection, were collected in c. $7 \mathrm{ml}$ of selective KL-T in $28-\mathrm{ml}$ screw-capped containers, kept in the refrigerator and periodically sent to the Tuberculosis Research Centre, Madras. During transport they were kept in insulated boxes without ice for $18-20 \mathrm{~h}$. A delay between resection and processing occurred in 105 (35\%) specimens which were received after 5-15 days. 


\section{Selective Kirchner's liquid medium}

The medium was prepared as follows [3]. Salt solution prepared with sodium hydrogen phosphate $19.0 \mathrm{~g}$, potassium dihydrogen phosphate $2.0 \mathrm{~g}$, magnesium sulphate $0.6 \mathrm{~g}$, sodium citrate $2.5 \mathrm{~g}$, casein hydrolysate $0.5 \mathrm{~g}$, glycerol $20 \mathrm{ml}$, phenol red $(0.4 \%$ solution $) 3 \mathrm{ml}$ in $1 \mathrm{~L}$ of distilled water was adjusted to $\mathrm{pH} 6.9$ and 7.2 and sterilised. Antibiotic solution was made with polymyxin B $31 \mathrm{mg}$, amphotericin B $10 \mathrm{mg}$, carbenicillin $100 \mathrm{mg}$ and trimethoprim $10 \mathrm{mg}$ in distilled water $10 \mathrm{ml}$. All four antibiotics were purchased from Sigma. These two solutions were mixed with sterile horse serum $100 \mathrm{ml}$ and distributed in universal containers.

\section{Culture methods}

The lymph node specimen was aseptically removed from KL-T and processed for culture of mycobacteria by standard procedures in multiple media [4]. The deposit of the ground homogenised specimen was decontaminated with $\mathrm{H}_{2} \mathrm{SO}_{4} 5 \%$, neutralised with distilled water and inoculated on to two sets of four media, i.e. Lowenstein-Jensen (LJ), LJ with sodium pyruvate $0.5 \%$ (LJ-P), selective oleic acid albumin agar (7H11) and Kirchner's liquid medium (KL). KL-T was also incubated after removal of the node and examined every week for any visible growth. Subcultures were made from KL and KL-T either at the end of the sixth week or as and when any growth was noticed. Cultures were identified based on results from the niacin and thermostable catalase tests and growth on LJ medium containing para-nitrobenzoic acid [4].

\section{Results and discussion}

Of the 297 lymph node specimens processed, 201 $(68 \%)$ yielded positive cultures (Table 1). This finding was similar to a report on childhood lymphadenitis, wherein the specimens were transported in plain bottles and processed on the same day, or within 1 or 2 days [1]. The delay in transportation of the specimens kept in KL-T did not adversely affect the culture positivity rate (Table 1). Of the 192 specimens received within 4 days, $134(70 \%)$ were positive by culture and of the 105 specimens received after 5-15 days, 67 (64\%) were positive. Thus, the rate of isolation in specimens received after 5-15 days was similar to that of specimens received within 4 days $(p=0.4)$. This finding is important, as Kulkarni [5] observed that the delay in transit of specimens had an adverse effect on culture positivity. In his study, M. tuberculosis was isolated from $59 \%$ of 101 lymph node specimens transported in sterile containers within 4 days and the yield was lower from the specimens that were processed on days $5-6(49 \%)$ or $7(40 \%)$. Similarly, Pamra and Mathur [6] also observed that $75 \%$ of 24 cervical nodes processed within 2 days were culture positive but this declined to $29 \%$ if the delay was $>7$ days.
Table 1. The delay in transportation of lymph node specimens and its influence on the rate of isolation

\begin{tabular}{|c|c|c|c|c|c|c|}
\hline \multirow{2}{*}{$\begin{array}{l}\text { Time taken } \\
\text { for transportation } \\
\text { (days) }\end{array}$} & \multirow{2}{*}{\multicolumn{2}{|c|}{$\begin{array}{l}\text { Number of } \\
\text { specimens }\end{array}$}} & \multicolumn{4}{|c|}{ Positive cultures } \\
\hline & & & \multicolumn{2}{|c|}{ No. } & \multicolumn{2}{|c|}{$\%$} \\
\hline $1-2$ & 73 & & 50 & & 68.5 & \\
\hline $3-4$ & 119 & & 84 & & 70.6 & \\
\hline $5-15$ & 105 & & 67 & & 63.8 & \\
\hline Total & 297 & & 201 & & 67.7 & \\
\hline
\end{tabular}

Table 2. Culture positivity in multiple media

\begin{tabular}{lcr}
\hline Media & Positive cultures (\%) \\
\hline KL-T & 77 & 38 \\
KL-T, LJ & 171 & 85 \\
KL-T, LJ, KL & 196 & 98 \\
KL-T, LJ, KL, LJP, 7H11 & 201 & 100 \\
\hline
\end{tabular}

Culture positivity rates obtained in KL-T and other media are presented in Table 2. Subcultures of KL-T on LJ yielded 77 (38\%) of 201 positive cultures. Thus, KL-T supports the growth of tubercle bacilli from lymph node specimens during storage and transport. It is important to note that the excised biopsy material was in contact with KL-T only during storage and transit. This period of contact with KL-T was enough to allow retrieval of 77 (38\%) 201 positive cultures: $58(30 \%)$ of 134 specimens in KL-T for $<5$ days and 19 (18\%) of 67 specimens for 5-15 days, were culture positive (not shown); the difference was not significant $(\mathrm{p}=0.06)$.

The number of culture positive results obtained on both KL-T and LJ accounted for $85 \%$ (Table 2) whereas $\mathrm{KL}-\mathrm{T}$ together with $\mathrm{LJ}$ and $\mathrm{KL}$ recovered as much as $98 \%$ of the total positive cultures. The use of LJ-P and more expensive media such as $7 \mathrm{H} 11$ did not contribute significantly to the primary isolation of tubercle bacilli. This finding compared well with earlier observations, thereby indicating that these media can be dispensed with for the primary isolation of tubercle bacilli from extrapulmonary specimens.

This study concludes that lymph node specimens can be stored at $4^{\circ} \mathrm{C}$ for up to 15 days and subsequently be transported at ambient temperature for $18-24 \mathrm{~h}$ in KL-T to a central laboratory without any loss of viability. This suggests that maintaining a cold chain during transit may be unnecessary.

We thank Mr S. Sivasubramanian, for his help in statistical analysis. The technical assistance of Mr G. Santhanakrishnan and Mrs N. S. Gomathi is gratefully acknowledged.

\section{References}

1. Jawahar MS, Sivasubramanian S, Vijayan VK et al. Short course chemotherapy for tuberculous lymphadenitis in children. BMJ 1990; 301: 359-362. 
2. Campbell IA, Dyson AJ. Lymph node tuberculosis: a comparison of treatments 18 months after completion of chemotherapy. Tubercle 1979; 60: 95-98.

3. Mitchison DA, Allen BW, Manickavasagar D. Selective Kirchner medium in the culture of specimens other than sputum for mycobacteria. J Clin Pathol 1983; 36: 1357-1361.

4. Paramasivan $\mathrm{CN}$, Vanajakumar, Alexander $\mathrm{C}$, Venkatesan $\mathrm{P}$
Somasundaram PR, Prabhakar R. Use of multiple media for the cultivation of mycobacteria from specimens other than sputum. Ind J Med Res 1987; 86: 290-294.

5. Kulkarni KG. Bacteriological study of tuberculous lymphadenitis. Ind $J$ Tuberc 1974; 21: 60-84.

6. Pamra SP, Mathur GP. A cooperative study of tuberculous cervical lymphadenitis. Ind $J$ Med Res 1974; 62: 1631-1646. 